\title{
INFLUÊNCIA DA QUANTIDADE DE AMÔNIO NA SÍNTESE DE NANOPARTÍCULAS DE ÓXIDO DE FERRO POR MICROEMULSÃO
}

\author{
Maria Tereza Cortez Fernandes e Elizabete Yoshie Kawachi* \\ Instituto Tecnológico de Aeronáutica, Praça Marechal Eduardo Gomes, 50, 12228-900 São José dos Campos - SP, Brasil
}

Recebido em 4/5/09; aceito em 1/2/10; publicado na web em 3/5/10

\begin{abstract}
THE INFLUENCE OF AMMONIUM QUANTITY ON THE SYNTHESIS OF IRON OXIDE NANOPARTICLES IN MICROEMULSION. Iron oxide nanoparticles were synthesized in microemulsion systems composed by Triton X-100/hexyl alcohol/ cyclohexane/aqueous solution. The nanoparticles were synthesized in microemulsions containing different amounts of ammonium, in order to evaluate the influence of this parameter on the size of the nanoparticles and on the phase transformation after heat treatment. Powder materials were obtained after centrifugation, washing and drying, and they were analyzed as synthesized and after heating at 350,500 and $1000^{\circ} \mathrm{C}$. It was observed that the higher amount of ammonium induced smaller particles and minor phase transformation, possibly due to a preferential nucleation process.
\end{abstract}

Keywords: nanoparticle; nucleation effect; iron oxide phase transformation.

\section{INTRODUÇÃO}

O desenvolvimento científico atual busca materiais em escala nanométrica, em função da possibilidade de obtenção de novas propriedades físicas e químicas, determinadas pelo reduzido tamanho (confinamento quântico) e pelo aumento da razão área/volume (efeitos de superfície). ${ }^{1}$ Desta maneira, diversos tipos de materiais nanoestruturados têm sido objeto de pesquisa, tais como metais ${ }^{2} \mathrm{e}$ ligas metálicas, ${ }^{3}$ cerâmicas em geral ${ }^{4}$ e óxidos. ${ }^{5}$ Entre estes materiais, nanopartículas de óxidos de ferro têm sido amplamente investigados devido ao diversificado campo de aplicações, como ferrofluidos, ${ }^{6}$ dispositivos de armazenamento de dados, ${ }^{7}$ contraste para imagem por ressonância magnética, ${ }^{8,9}$ carregador de fármacos,${ }^{8,10}$ catálise,${ }^{11}$ etc.

Várias técnicas de síntese de óxidos de ferro nanoparticulados têm sido propostas, como alternativa ao método tradicional de moagem, como sol-gel, ${ }^{12}$ coprecipitação, ${ }^{13}$ Pechini,${ }^{14}$ aerosol, ${ }^{15}$ combustão,,${ }^{11,16}$ sonoquímico ${ }^{17}$ e microemulsão. ${ }^{18}$ Cada um destes métodos tem vantagens e desvantagens que resultam em materiais mais ou menos homogêneos em relação à composição química, tamanho e forma das partículas. Neste trabalho, as nanopartículas de óxido de ferro foram obtidas por microemulsão, cujo diferencial está no fato de que as partículas formadas apresentam dimensões dentro de uma faixa de tamanhos estreita, o que limita o número de variáveis a serem consideradas no trabalho. Outra vantagem do método está relacionada ao baixo custo da aparelhagem empregada para a preparação do material. Como as condições de síntese influenciam fortemente as características físicas e químicas dos materiais e, portanto, as propriedades dos mesmos, a síntese dos óxidos de ferro foi realizada estabelecendo como parâmetro de controle a quantidade de hidróxido de amônio no sistema e avaliando a influência deste no tamanho das partículas e na evolução das transições de fase do material, submetido a tratamento térmico até $1000{ }^{\circ} \mathrm{C}$.

\section{Síntese de nanopartículas por microemulsão}

As microemulsões são sistemas coloidais, assim como o sol-gel e o aerosol, cuja característica básica é a dispersão de uma fase em outra. No caso da microemulsão, estas fases são líquidas, sendo

\footnotetext{
*e-mail: bete@ita.br
}

uma aquosa e uma orgânica. Para manter a estabilidade deste tipo de sistema, é necessária a presença de uma substância anfifílica ou surfatante e, muitas vezes, de um coadjuvante para a surfatação, chamado de cosurfatante, formando sistemas ternários e pseudoternários, respectivamente.

Devido a interações intermoleculares, moléculas da fase aquosa tendem a se isolar da fase orgânica e isto ocorre com a interposição de moléculas de surfatante, originando os nanorreatores, dentro dos quais pode ocorrer uma reação química promovendo a geração de nanopartículas. Para tanto, os materiais de interesse são sintetizados a partir de reagentes precursores solubilizados na fase aquosa, sob agitação vigorosa, possibilitando a troca de matéria por colisão e coalescência dos nanorreatores. ${ }^{19,20}$

Neste trabalho foi utilizado um sistema pseudoternário formado por Triton X-100 como surfatante, hexanol como cosurfatante, ciclohexano como fase oleica ou dispersante, soluções salinas de íons ferro como fase dispersa e hidróxido de amônio como agente precipitante.

\section{PARTE EXPERIMENTAL}

As nanopartículas de óxido de ferro foram obtidas em microemulsão, contendo quantidades distintas de hidróxido de amônio. Os sistemas de síntese foram obtidos a partir da mistura de duas microemulsões básicas, denominadas MEa e MEb, as quais diferiam apenas na composição da fase aquosa. A mistura MEa envolveu os seguintes reagentes: Triton X-100 (P.A., Merk), hexanol (P.A., Merk), ciclohexano (P.A., Vetec) e uma solução aquosa contendo $0,1 \mathrm{~mol} / \mathrm{L}$ de $\mathrm{Fe}^{2+}$ e $0,15 \mathrm{~mol} / \mathrm{L}$ de $\mathrm{Fe}^{3+}$ (razão molar $\mathrm{Fe}^{3+} / \mathrm{Fe}^{2+}=1,5$ ), preparada a partir dos sais $\mathrm{FeCl}_{2} \cdot 4 \mathrm{H}_{2} \mathrm{O}$ (P.A., Merk) e $\mathrm{FeCl}_{3} \cdot 6 \mathrm{H}_{2} \mathrm{O}$ (P.A., Synth). A microemulsão foi preparada adicionando os reagentes sob agitação magnética e a temperatura ambiente. Na mistura MEb, empregou-se como fase aquosa uma solução a $25 \%(\mathrm{~m} / \mathrm{m})$ em NH $\mathrm{NH}_{4} \mathrm{OH}$ (P.A., Merck). As composições dos sistemas MEa e MEb são apresentadas na Tabela 1.

Para a síntese dos óxidos de ferro, as microemulsões MEa e $\mathrm{MEb}$ foram misturadas em diferentes quantidades. A microemulsão ME10 foi obtida adicionando-se $10 \mathrm{~mL}$ de MEb a $129 \mathrm{~mL}$ de MEa, já a microemulsão ME20 foi obtida adicionando-se $20 \mathrm{~mL}$ de $\mathrm{MEb}$ ao mesmo volume de MEa.

Em ambos os casos, a microemulsão MEb foi adicionada gota a gota à MEa, sob agitação magnética, de modo a possibilitar a colisão, 
Tabela 1. Composição das microemulsões utilizadas na síntese dos óxidos de ferro

\begin{tabular}{llc}
\hline Sistema & Reagente & Concentração (\% m) \\
\hline \multirow{4}{*}{ MEa } & Triton X-100 & 23,8 \\
& Ciclo-hexano & 56,2 \\
& Hexanol & 7,4 \\
& $\mathrm{Fe}^{2+} / \mathrm{Fe}^{3+}(\mathrm{aq})$ & 12,6 \\
& Triton X-100 & 24,1 \\
$\mathrm{MEb}$ & Ciclo-hexano & 56,8 \\
& Hexanol & 7,5 \\
& $\mathrm{NH}_{4} \mathrm{OH}(\mathrm{aq})$ & 11,6 \\
\hline
\end{tabular}

coalescência e troca de matéria entre os nanorreatores contendo as soluções de íons ferro e a solução de hidróxido de amônio. Nestas condições, ocorre uma reação de coprecipitação que pode ser representada pela Equação 1:

$\mathrm{Fe}^{2+}(\mathrm{aq})+2 \mathrm{Fe}^{3+}(\mathrm{aq})+8 \mathrm{OH}^{-}(\mathrm{aq}) \rightarrow \mathrm{Fe}_{3} \mathrm{O}_{4}(\mathrm{~s})+4 \mathrm{H}_{2} \mathrm{O}(\mathrm{l})$

As microemulsões foram deixadas em repouso por 3 semanas, à temperatura ambiente. Após este período, os sólidos formados foram separados das misturas por centrifugação a $3000 \mathrm{rpm}$, lavados três vezes com acetona (P.A., Quimex) e álcool etílico (P.A., Quimex) e foram secos ao ar. As amostras secas foram tratadas termicamente a 350,500 e $1000{ }^{\circ} \mathrm{C}$ por $1 \mathrm{~h}$ e identificadas como A10, A10-350, A10-500 e A10-1000 com referência, respectivamente, às amostras sintetizadas na microemulsão ME10 sem tratamento térmico e tratadas nas temperaturas indicadas. O mesmo critério de nomenclatura foi adotado para as amostras sintetizadas em ME20.

As partículas de óxido de ferro sintetizadas por microemulsão foram caracterizadas usando-se diferentes técnicas. A espectroscopia na região do infravermelho com transformada de Fourier (FT-IR) foi utilizada procurando destacar o comportamento da fase preferencial de óxido de ferro obtida pelo método da microemulsão. As amostras sintetizadas e tratadas termicamente a 350,500 e $1000{ }^{\circ} \mathrm{C}$ foram analisadas empregando-se a técnica de pastilha de $\mathrm{KBr}$, na região de 4000 a $400 \mathrm{~cm}^{-1}$ (destacando-se neste trabalho apenas a região de 2000 a $380 \mathrm{~cm}^{-1}$ referente à região de absorção da ligação $\mathrm{Fe}-\mathrm{O}$ ), com resolução de $4 \mathrm{~cm}^{-1}$ e 20 varreduras. O equipamento empregado foi o Spectrum One da Perkin Elmer.

Análise de difração de raios X (DRX) foi realizada nas amostras tratadas a $500{ }^{\circ} \mathrm{C}$, utilizando o difratômetro de raios X Philips Modelo 1840/1830, com fonte de radiação $\operatorname{CuK\alpha }(\lambda=1,541837 \AA ̊ \Omega)$ gerada a $40 \mathrm{kV}$ e $25 \mathrm{~mA}$ e dotado de filtro de níquel e fenda de 0,2 $\mathrm{mm}$. As análises foram realizadas no intervalo de $20^{\circ} \leq 2 \theta \leq 80^{\circ}$, com incrementos $\Delta \theta=0,017^{\circ}$, a uma velocidade de varredura de $0,030^{\circ} / \mathrm{s}$. Os dados obtidos foram submetidos a um filtro de média móvel com ponderação de Hamming ${ }^{21}$ que enfatiza os picos, fazendo a rejeição do ruído de alta frequência presente no difratograma original. Este procedimento possibilitou a definição da posição angular $2 \theta$ em que ocorrem os picos mais significativos do difratograma.

O tamanho médio (D) de cristalito das amostras foi determinado a partir dos dados de DRX originais, aplicando-se a Equação de Debye-Scherrer:

$$
D=\frac{0,9 \lambda}{\beta \cos \theta}
$$

onde $\beta$ é a abertura (em radianos) a meia altura do pico mais intenso e característico da fase cristalina do material. A abertura de um pico é normalmente considerada como a abertura de uma função Gaussiana que se ajusta ao difratograma nas vizinhanças do pico considerado. Neste trabalho, avaliou-se o tamanho dos cristalitos de magemita, a partir da abertura a meia altura do pico situado em $2 \theta$ $\approx 35,6^{\circ}$, cuja posição absoluta foi determinada previamente a partir dos dados filtrados.

As amostras tratadas a $500{ }^{\circ} \mathrm{C}$ foram caracterizadas quanto a sua morfologia por microscopia eletrônica de varredura (MEV) e microscopia de força atômica (AFM). Para a análise por MEV, as amostras foram depositadas diretamente sobre fita de carbono e posteriormente metalizadas com ouro. A análise foi realizada em um equipamento Jeol modelo JSM - 6360LV, operando em $15 \mathrm{kV}$. Para a análise por AFM, uma pequena porção de cada amostra foi suspensa em isopropanol e levada ao banho de ultrassom por $15 \mathrm{~min}$. Uma alíquota de cada suspensão foi depositada sobre um substrato de silício previamente limpo. A análise de AFM foi realizada utilizando um equipamento Shimadzu modelo SPM 9500 módulo Minus K.

\section{RESULTADOS E DISCUSSÃO}

A formação de microemulsão foi avaliada visualmente, verificando-se que a mistura das microemulsões MEa e MEb (transparentes) resultou em sistemas monofásicos, translúcidos e marrons, os quais permaneceram estáveis durante 3 semanas. A coloração marrom sugere a formação de magemita $\left(\gamma-\mathrm{Fe}_{2} \mathrm{O}_{3}\right)$. Já a translucidez e a estabilidade dos sistemas revelam a formação de partículas de dimensões coloidais.

O método da microemulsão foi escolhido para a síntese dos óxidos de ferro por resultar em partículas de dimensões dentro de uma faixa estreita de tamanhos, de modo que a variação granulométrica pudesse ser atribuída exclusivamente aos parâmetros empregados na síntese. Além do tamanho das partículas, outra variável estudada foi a fase cristalina do óxido sintetizado e submetido a tratamento térmico. As fases mais comuns são a magnetita $\left(\mathrm{Fe}_{3} \mathrm{O}_{4}\right)$, a magemita $\left(\gamma-\mathrm{Fe}_{2} \mathrm{O}_{3}\right)$ e a hematita $\left(\alpha-\mathrm{Fe}_{2} \mathrm{O}_{3}\right)$. As temperaturas nas quais normalmente se observam as conversões entre estas fases são mostradas na Equação $3 .{ }^{22}$

$\mathrm{Fe}_{3} \mathrm{O}_{4} \stackrel{200-400^{\circ} \mathrm{C}}{\longrightarrow} \gamma-\mathrm{Fe}_{2} \mathrm{O}_{3} \stackrel{400-550^{\circ} \mathrm{C}}{\longrightarrow} \alpha-\mathrm{Fe}_{2} \mathrm{O}_{3}$

Para a avaliação da composição cristalina dos óxidos de ferro sintetizados por microemulsão foram empregadas técnicas convencionais de caracterização de materiais, como FT-IR e DRX. Tanto as bandas de absorção no infravermelho quanto os picos de difração de raios $\mathrm{X}$ dos óxidos de ferro estão caracterizadas na literatura, ${ }^{23}$ porém a interpretação destes sinais se torna difícil quando mais de uma fase coexiste na amostra. Esta mistura de fases ocorre quando a síntese se processa em atmosfera oxidante e, especialmente, durante o tratamento térmico em atmosfera ambiente.

Os espectros de FT-IR das amostras obtidas a partir das microemulsões ME10 e ME20 não submetidas a tratamento térmico e tratadas a 350,500 e $1000{ }^{\circ} \mathrm{C}$ são mostrados na Figura 1. Nesta Figura, observa-se a presença de bandas na região entre 1550 e $800 \mathrm{~cm}^{-1}$ para as amostras sem tratamento térmico e tratadas a $350{ }^{\circ} \mathrm{C}$. Estas bandas caracterizam a presença de compostos orgânicos, especialmente do surfatante, identificado pelas bandas de vibração de grupos fenol, $-\mathrm{CH}_{2},-\mathrm{CH}_{3}$ e $\left(\mathrm{H}_{2}\right) \mathrm{C}-\mathrm{O}$. A partir de $500{ }^{\circ} \mathrm{C}$, estas bandas não são mais observadas. A presença destas bandas indica que moléculas do surfatante permaneceram adsorvidas na superfície das amostras sintetizadas, mesmo após o processo de lavagem, sendo eliminadas durante o tratamento térmico entre 350 e $500{ }^{\circ} \mathrm{C}$.

Na região entre 700 e $380 \mathrm{~cm}^{-1}$, os espectros de FT-IR das amostras A10 e A20 sem tratamento térmico e tratadas a 350 e $1000{ }^{\circ} \mathrm{C}$ apresentam as mesmas bandas, indicando que não há diferença de composição nestas amostras. Por outro lado, as amostras tratadas a $500{ }^{\circ} \mathrm{C}$ apresentam pequenas diferenças que apontam para a presença de fases distintas. Para as amostras sem tratamento térmico, observase a presença de magnetita e magemita, representadas pelas bandas em 444 e $564 \mathrm{~cm}^{-1}$, respectivamente. Com o tratamento térmico a 


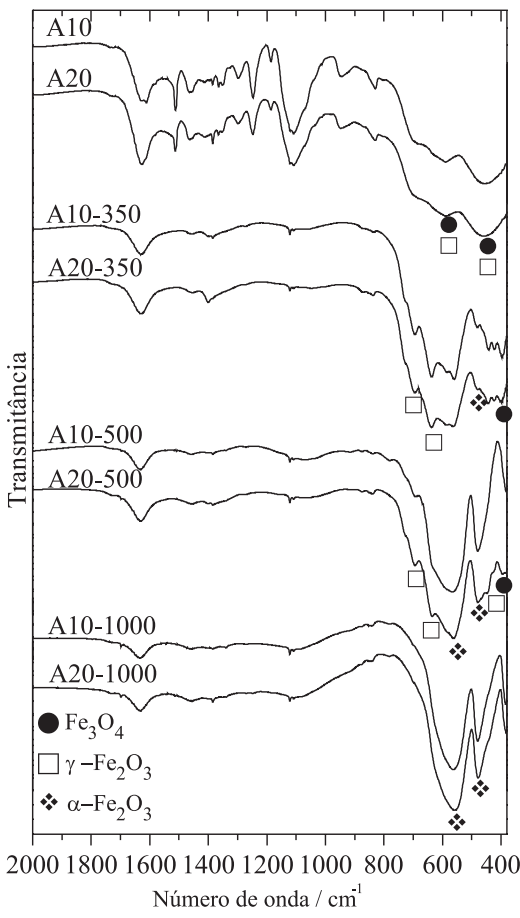

Figura 1. Espectros de FT-IR das amostras A10 (a) e A20 (b), sem tratamento térmico e tratadas a 350,500 e $1000{ }^{\circ} \mathrm{C}$

$350{ }^{\circ} \mathrm{C}$ verifica-se a presença de bandas que podem ser atribuídas à magnetita, magemita e hematita, tanto para a amostra sintetizada na microemulsão ME10 quanto na ME20. O comportamento das amostras se diferencia após o tratamento a $500{ }^{\circ} \mathrm{C}$. Para a amostra A20-500, além das bandas em 447 e $640 \mathrm{~cm}^{-1}$, relacionadas à hematita e também observadas na amostra A10-500, ainda se observam pequenas bandas em 481 e $562 \mathrm{~cm}^{-1}$ atribuídas à magemita. As fases identificadas nos espectros de FT-IR para as amostras tratadas a diferentes temperaturas são indicadas na Tabela 2.

Tabela 2. Composição das amostras tratadas a $500{ }^{\circ} \mathrm{C}$ e estimativa do tamanho de cristalito determinado por Debey-Scherrer a partir do pico $2 \theta \approx 35,7^{\circ}$

\begin{tabular}{lcc}
\hline Amostra & Composição & Tamanho/nm \\
\hline A10 & $\mathrm{M}+\gamma$ & - \\
A20 & $\mathrm{M}+\gamma$ & - \\
A10-500 & $\alpha+\gamma$ & 31,2 \\
A20-500 & $\gamma+\alpha+\mathrm{M}$ & 29,4 \\
\hline
\end{tabular}

$\mathrm{M}=$ magnetita; $\gamma=$ magemita; $\alpha=$ hematita

Os resultados de FT-IR indicam que a amostra A10 apresenta temperatura da transição $\gamma-\mathrm{Fe}_{2} \mathrm{O}_{3} \rightarrow \alpha-\mathrm{Fe}_{2} \mathrm{O}_{3}$ menor que a amostra A20, indicando a menor estabilidade térmica da amostra A10. A Figura 2 apresenta os difratogramas de raios $\mathrm{X}$ destas amostras tratadas a $500{ }^{\circ} \mathrm{C}$, para os quais se observa uma mistura de fases $\gamma$ e $\alpha-\mathrm{Fe}_{2} \mathrm{O}_{3}$. No entanto, pode-se observar a diferença de intensidade dos picos característicos de cada fase, sendo que a amostra A10-500 apresenta os picos característicos de hematita $\left[33,1^{\circ}(104) ; 35,6^{\circ}(110)\right.$; $54,0^{\circ}(116) ; 24,1^{\circ}(012)$ e $\left.49,4^{\circ}(024)\right]$ em maior evidência, com o pico em $33,2^{\circ}$ mais intenso que o pico em $35,7^{\circ}$, enquanto a amostra A20500 apresenta tanto os picos característicos de magemita $\left[35,7^{\circ}(311)\right.$; $30,3^{\circ}(220)$ e $\left.62,9^{\circ}(440)\right]$ quanto os de hematita em evidência; no entanto, os picos em $33,2^{\circ}$ e em $35,7^{\circ}$ apresentam relação de intensidade inversa em relação à amostra A10-500. Isto indica que a magemita foi majoritariamente transformada em hematita na amostra A10 tratada a $500{ }^{\circ} \mathrm{C}$, o mesmo não sendo observado na amostra
A20 tratada à mesma temperatura, resultado que corrobora com as análises por FT-IR. A presença de magnetita não pode ser confirmada por esta técnica, já que a magnetita e a magemita apresentam padrão cristalográfico semelhante.

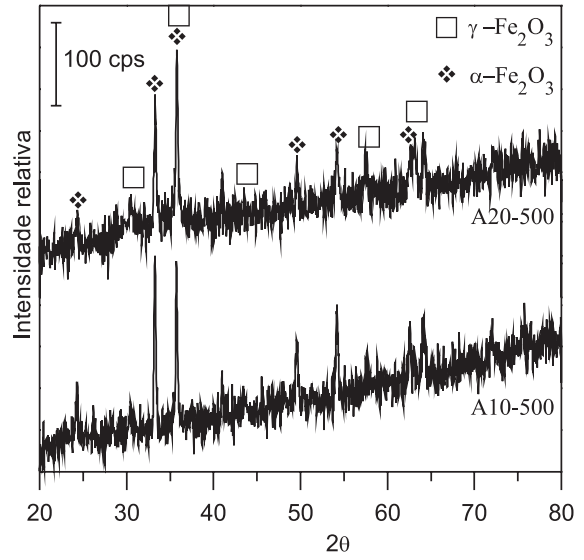

Figura 2. DRX das amostras A10 e A20 tratadas a $500{ }^{\circ} \mathrm{C}$

A princípio, a síntese em microemulsão ocorre de forma análoga à síntese em sistema aquoso, com o diferencial da limitação física imposta pela interface formada pelas moléculas de surfatante. Em sistemas aquosos convencionais, o tamanho das partículas sintetizadas diminui em função do excesso de um dos reagentes, ${ }^{19,24}$ pois a nucleação é favorecida em relação ao crescimento dos cristais. Neste trabalho, utilizaram-se quantidades grandes de $\mathrm{NH}_{4} \mathrm{OH}$ na síntese em microemulsão, o que pode ter contribuído não só para o favorecimento do processo de nucleação, como também para uma maior eficiência dos eventos relacionados especificamente à síntese em microemulsão, principalmente a troca intermicelar. Neste aspecto, quanto maior a quantidade de reagentes, maior é a probabilidade de colisão entre nanorreatores contendo as espécies reacionais e, portanto, maior a eficiência do sistema de síntese. Além disso, a difusão de vapor de $\mathrm{NH}_{3}$ na microemulsão e sua pequena (porém não desprezível) solubilidade na fase orgânica favorecem o processo de nucleação e promovem a formação de partículas de menor tamanho. O tamanho médio de cristalito das amostras A10-500 e A20-500 foi estimado por Debye-Sherrer e os valores estão apresentados na Tabela 2. Verifica-se que a amostra A20-500 apresenta cristalitos menores que a amostra A10-500.

Neste ponto, cabe destacar a influência do tratamento térmico no comportamento químico e morfológico dos óxidos de ferro sintetizados em microemulsão com diferentes quantidades de amônio. De acordo com as análises de FT-IR e DRX, o comportamento químico das amostras tratadas a $500{ }^{\circ} \mathrm{C}$ foi relativamente diferente, indicando que a maior quantidade de amônio favorece a estabilidade térmica do óxido. Em relação ao tamanho das partículas, é de se esperar que o aquecimento induza o crescimento (por difusão atômica) e a cristalização (por rearranjo atômico). A estimativa do tamanho de cristalito das amostras tratadas a $500{ }^{\circ} \mathrm{C}$ foi realizada, levando-se em consideração o pico característico da magemita, a $2 \theta \quad 35,7^{\circ}$. No entanto, cristais de hematita também apresentam pico de difração relativamente intenso nesta região e, devido ao aquecimento, a hematita apresenta maior cristalinidade que a magemita. Desta forma, é possível que os tamanhos de cristalito estimados sejam referentes aos cristais de hematita, subestimados devido a um alargamento causado pela sobreposição do pico da magemita. Análises microscópicas podem auxiliar na caracterização destas amostras.

A análise por AFM mostrou que as partículas obtidas por microemulsão e tratadas termicamente a $500{ }^{\circ} \mathrm{C}$ apresentam dimensões 
nanométricas, conforme pode ser observado na Figura 3. As partículas analisadas por AFM apresentam dimensões menores que as estimadas por Debye-Scherrer, no entanto, esta discrepância pode ser atribuída à suposição levantada no parágrafo anterior, em que se estimou o tamanho de cristalito da hematita, enquanto as partículas observadas no AFM podem representar as partículas menores de magemita que ainda não foram convertidas à hematita e que foram desaglomeradas por ação do banho de ultrassom. Comparando as barras de escala das micrografias de AFM, observa-se dimensões médias menores para a amostra A20-500 (Figura 3b) que para a amostra A10-500 (Figura 3a).
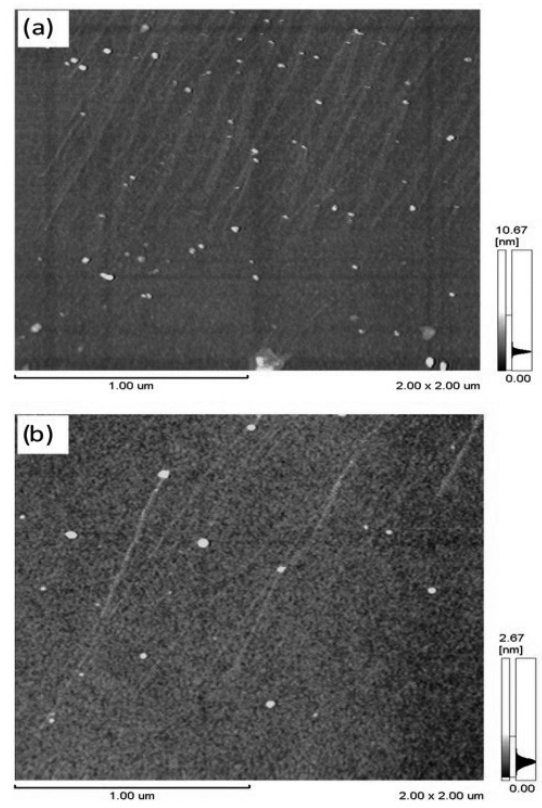

Figura 3. Micrografias de AFM das amostras A10 (a) eA20(b) tratadas a $500^{\circ} \mathrm{C}$

As análises por MEV não possibilitaram a observação das partículas individuais. No entanto, é possível observar nas micrografias da Figura 4 que a quantidade de amônio utilizada nas sínteses por microemulsão influenciou no tamanho e no grau de aglomeração das partículas das amostras tratadas a $500{ }^{\circ} \mathrm{C}$. Observa-se que a amostra A10-500 (Figura 4a) apresentou partículas maiores (de até $1 \mu \mathrm{m}$ ) que a amostra A20-500 (Figura 4b), cujas partículas não ultrapassam 0,5 $\mu \mathrm{m}$. Estas partículas apresentam arestas bem definidas e sua morfologia pode ser associada ao arranjo romboédrico dos cristais de hematita, o que concorda com os dados de DRX, nos quais se observou um padrão cristalino de hematita, com partículas subestimadas de aproximadamente $30 \mathrm{~nm}$. Além disso, em ambos os casos, as amostras apresentam pequenos aglomerados de partículas sem morfologia definida que podem representar partículas da fase magemita, cujas partículas individuais podem ter sido identificadas por AFM.

A diferença de tamanho de partícula da hematita nas amostras A10-500 e A20-500 poderia ser interpretada como sendo o resultado do menor ou maior grau de conversão de magemita a hematita, respectivamente. No entanto, ao sinterizar estas amostras a $1000{ }^{\circ} \mathrm{C}$, observa-se por FT-IR que ambas apresentam apenas hematita em sua composição, porém as partículas observadas por MEV (Figura 1S, material suplementar) continuam mostrando a mesma tendência de tamanhos, ou seja, menor tamanho de partícula para a amostra A20 e maior para a A10, indicando que a quantidade de amônio utilizada na síntese do óxido de ferro em microemulsão tem grande influência tanto na estabilidade térmica do material, quanto no tamanho das partículas, mesmo após tratamento térmico.
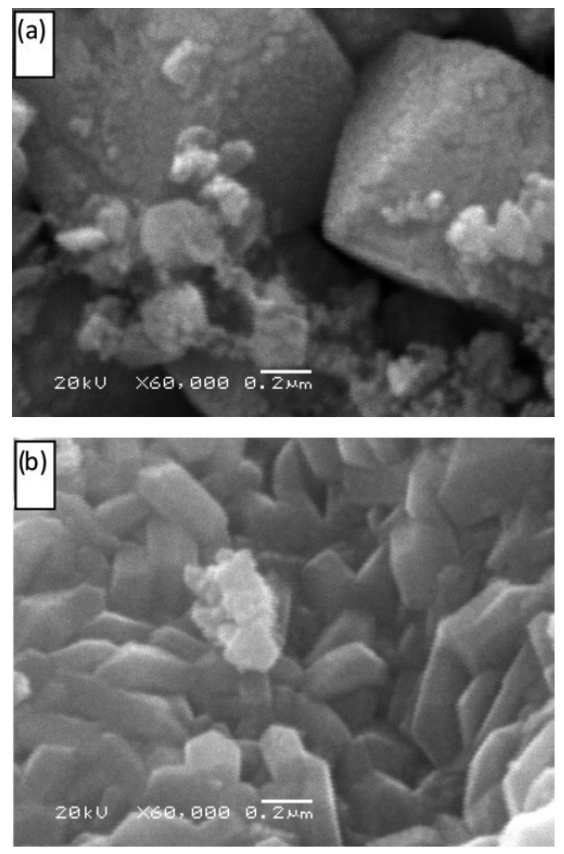

Figura 4. Micrografias de MEV das amostras A-10(a) e A20(b) tratadas a $500^{\circ} \mathrm{C}$

Verifica-se, portanto, que o tamanho das partículas primárias obtidas diretamente do processo de síntese interfere na forma como elas serão aglomeradas e, portanto, influenciando a morfologia e o tamanho das partículas submetidas a tratamento térmico.

\section{CONCLUSÕES}

A variação da quantidade de $\mathrm{NH}_{4} \mathrm{OH}$ na síntese de óxido de ferro por microemulsão influenciou tanto a estabilidade térmica em relação às transições de fase quanto o tamanho das partículas durante o tratamento térmico, sendo verificado que a amostra sintetizada na presença de maior quantidade de amônio (amostra A20) apresentou partículas de tamanhos menores e maior estabilidade que as partículas obtidas em menor quantidade de amônio (amostra A10). Após aquecimento a $500{ }^{\circ} \mathrm{C}$, a amostra A20 apresentou cristais de hematita de dimensões abaixo de $0,5 \mu \mathrm{m}$ com a presença de magemita com menor intensidade cristalográfica no DRX, enquanto a amostra A10 apresentou cristais de hematita de até $1 \mu \mathrm{m}$ com contribuição de magemita quase imperceptível no DRX. Esta tendência pode ser resultado da maior velocidade de nucleação, em detrimento do crescimento das partículas sintetizadas quando a quantidade de amônio por unidade de volume da microemulsão é maior.

\section{AGRADECIMENTOS}

Ao LAS/INPE pelas análises de DRX, ao IQ/Unicamp pelas análises de MEV, ao IEFF/ITA pelas análises de AFM, ao IAE/CTA pelas análises de FTIR e ao CNPq pelo apoio financeiro (Processo 475971/2007-8). M. T. C. Fernandes agradece à CAPES pela bolsa de mestrado.

\section{REFERÊNCIAS}

1. Duran, N.; Mattoso, C. H. L.; Morais, C. P.; Nanotecnologia, $1^{\mathrm{a}}$ ed, Artliber: São Paulo, 2006; Nathani, H.; Misra, R. D. K.; Mater. Sci. Eng., B 2004, 113, 228.

2. Pal, A.; Shah, S.; Devi, S.; African Physical Review 2007, 1, 1; Semagina, N.; Renken, A.; Laub, D.; Kiwi-Minsker, L.; J. Catal. 2007, 246, 308. 
3. Weihua, W.; Xuelin, T.; Kai, C.; Gengyu, C.; Colloids Surf., A 2006, 273,3 .

4. Carr, C. S.; Shantz, D. F.; Microporous Mesoporous Mater. 2005, 85, 284; Osseo-Asare, K.; Arriagada, F. J.; Colloids Surf. 1990, 50, 321; Kawachi, E. Y.; Tese de Doutorado, Universidade Estadual de Campinas, Brasil, 2002.

5. Pillai, V.; Shah, D. O.; J. Magn. Magn. Mater. 1996, 163, 243; Lee, Y., Lee, J.; Bae, C. J.; Park, J. G.; Noh, H. J.; Park, J. H.; Hyeon, T.; Adv. Funct. Mater. 2005, 15, 503.

6. Odenbach, S.; Colloids Surf, A 2003, 217, 171; Makovec, D.; Kosak, A.; Znidarsic, A.; Drofenik, N.; J. Magn. Magn. Mater. 2005, 289, 32; Bocanegra-Diaz, A.; Mohallem, N. D. S.; Sinisterra, R. D.; J. Braz. Chem. Soc. 2003, 14, 936.

7. Muhammed, M.; Tsakalakos, T.; J. Korean Ceram. Soc. 2003, 40, 1027.

8. Camilo, L. R.; Tese de Doutorado, Universidade de São Paulo, Brasil, 2006.

9. Laconte, L.; Nitin, N.; Bao, G.; Nanotoday 2005, 32.

10. Kim, E. H.; Lee, S. H.; Kwak, K. B.; Kim, K. B.; J. Magn. Magn. Mater. 2005, 289, 328.

11. Lula, R. P. T.; Melo, P. S.; Barros, B. S.; Gama, L.; Mariano, W. A.; Kiminami, R. H. G. A.; Costa, A. C. F. M.; Cerâmica 2005, 1, 102.

12. Yue, Z.; Guo, W.; Zhou, J.;Gui, Z.; Li, L.; J. Magn. Magn. Mater. 2003, 270, 216; Lima, P. T.; Bertran, C. A.; Thim, G. P.; Quim. Nova 1998, 21, 608; Park, S. K.; Kim, K. D.; Kim, H. T.; Colloids Surf., A 2002, 197, 7.

13. Jiang, W.; Yang, H. C.; Yang, S. Y.; Horing, H. E.; Hung, J. C.; Chen, Y. C.; Hong, C. Y.; J. Magn. Magn. Mater. 2004, 283, 210; AyalaValenzuela, O.; Matutes-Aquino, J.; Betancourt-Galindo, R.; GarciaCerda, L. A.; Fernandez, O. R.; Fannin, P. C.; Giannitsis, A. T.; J. Magn. Magn. Mater. 2005, 294, e37.
14. Maciel, A. P.; Longo, E.; Leite, E. R.; Quim. Nova 2003, 26, 1; Candeia, R. A.; Souza, M. A. F.; Bernardi, M. I. B.; Maestrelli, S. C.; Santos, I. M. G.; Souza, A. G.; Longo, E.; Ceram. Int. 2007, 33, 521.

15. Kim, D.; Vasilieva, E. S.; Nasibulin, A. G.; Lee, T. W.; Tulochko, O. V.; Kim, B. K.; Materials Science Forum 2007, 534-536, 9; Segal, D.; J. Mater. Chem. 1997, 7, 1297.

16. Costa, A. C. F. M., Gama, L.; Portela, F. A.; Silva, V. J.; Torquato, R. A.; Kiminami, R. H. G. A.; Paiva-Santos, C. O.; Oliveira, J. B. L.; Revista Eletrônica de Materiais e Processos 2007, 2, 10.

17. Gedanken, A.; Curr. Sci. 2003, 85, 1720; Kesavan, V.; Dhar, D.; Koltypin, Y.; Perkas, N.; Palchik, O.; Gedanken, A.; Chandrasekaran, S.; Pure Appl. Chem. 2001, 73, 85.

18. Pillai, V.; Kumar, P.; Hou, N. J.; Ayyub, P.; Shah, D. O.; Adv. Colloid Interface Sci. 1995, 55, 241; López-Quintela, M. A.; Tojo, C.; Blanco, N. C.; Garcia Rio, L.; Leis, J. R.; Curr. Opin. Colloid Interface Sci. 2004, 9, 264; Pileni, M. A.; Langmuir 1997, 13, 3266.

19. Kumar, A. R.; AIChE J. 2003, 50, 1556.

20. Sato, H.; Tsubaki, Y.; Hirai, T.; Komasawa, I.; Ind. Eng. Chem. Res. 1997, 96, 92.

21. Bozic, S. M.; Digital and Lalman filtering, Edward Arnold: Londres, 1970.

22. Lepp, H.; Am. Mineral. 1957, 42, 679.

23. Bruce, I. J.; Taylor, J.; Todd, M.; Davies, M. J.; Botioni, E.; Sangregori, C.; Sen, T.; J. Magn. Magn. Mater. 2004, 284, 145.

24. Lopéz-Quintela, M. A.; Curr. Opin. Colloid Interface Sci. 2003, 8, 137; Fu, X.; Qutubuddin, S.; Colloids Surf., A. 2001, 179, 65; Tojo, C.; Barroso, F.; de Dios, M.; J. Colloid Interface Sci. 2006, 296, 591. 


\section{INFLUÊNCIA DA QUANTIDADE DE AMÔNIO NA SÍNTESE DE NANOPARTÍCULAS DE ÓXIDO DE FERRO} POR MICROEMULSÃO

Maria Tereza Cortez Fernandes e Elizabete Yoshie Kawachi*

Instituto Tecnológico de Aeronáutica, Praça Marechal Eduardo Gomes, 50, 12228-900 São José dos Campos - SP, Brasil

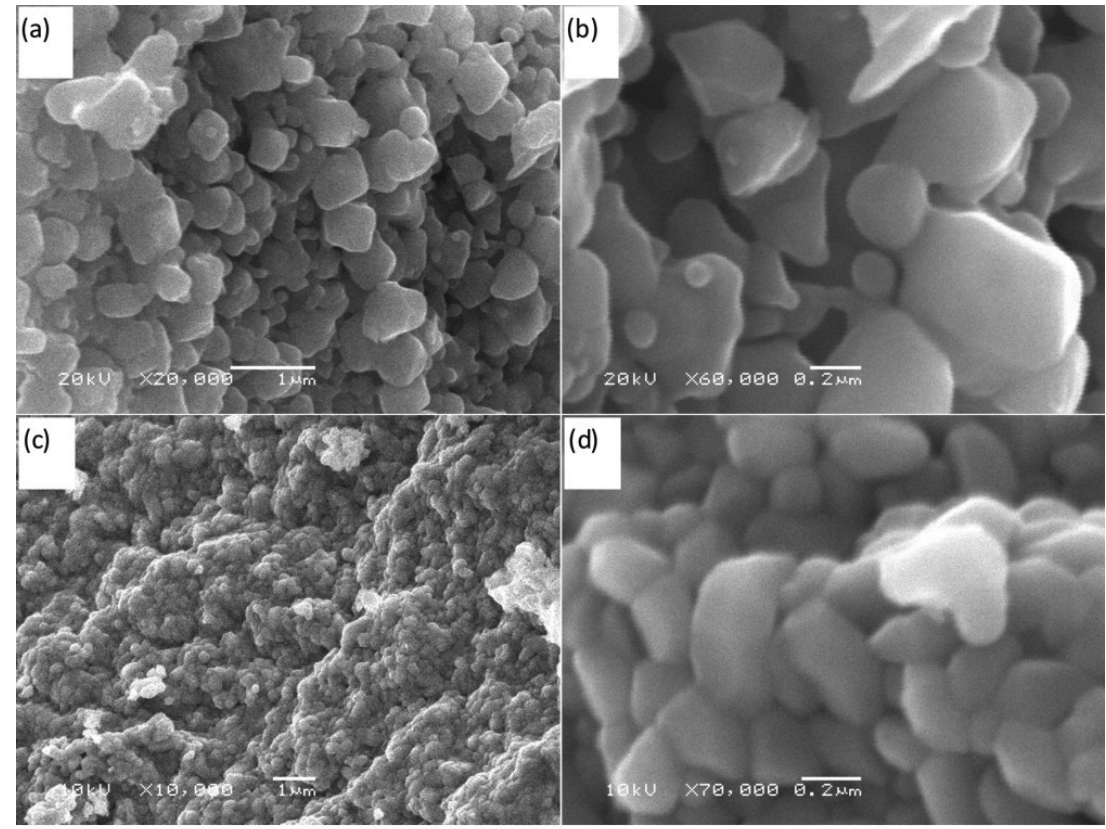

Figura 1S. Micrografias de MEV das amostras A10 $(a, b)$ e $A 20(c, d)$, tratadas a $1000{ }^{\circ} \mathrm{C}$ 